Case Report:

\title{
COVID-19 Infection Leading to Lethal Spondylodiscitis with Spinal Abscess
}

\author{
Soheil Naderi ${ }^{1}$ (D) Nima Ostadrahimi ${ }^{1}$ (D) Abdol Reza Sheikhrezaei ${ }^{1}$ (D) , Daniel Hänggi ${ }^{2}$ (D, Sajjad Muhammad ${ }^{2 *}$ (C) \\ 1. Department of Neurosurgery, Imam Khomeini Hospital, Tehran University of Medical Sciences, Tehran, Iran \\ 2. Department of Neurosurgery, Medical Faculty, Heinrich-Heine-University, Düsseldorf, Germany
}

$\begin{aligned} & \text { Use your device to scan } \\ & \text { and read the article online }\end{aligned}$
lodiscitis with Spinal Abscess. Iran J Neurosurg. 2020; 6(3):151-154. http://dx.doi.org/10.32598/irjns.6.3.6

(c) (1) (5)

Article info:

Received: 10 Mar 2020

Accepted: 13 May 2020

Available Online: $01 \mathrm{Jul} 2020$

Keywords:

COVID-19, Neurosurgery,

Neurological complications, Spondylodiscitis

\section{ABSTRACT}

Background and Importance: Secondary complications after SARS-CoV-2 virus infection including cardiovascular and neurological pose additional morbidity and mortality. The data on spondylodiscitis with spinal abscess after COVID-19 infection is very limited.

Case Presentation: A 71-year-old man presented with tachypnea and cough since 24 hours before admission. He was febrile and his chest CT showed a typical picture of bilateral covid-19 pneumonia. Nasopharyngeal swabs showed a positive PCR for COVID-19. The patient was admitted to the Intensive Care Unit (ICU) due to low oxygen saturation. Forty-eight hours after admission, the patient started to develop paraparesis which rapidly progressed to paraplegia within a few hours. In the MRI scan, multilevel spondylodiscitis and epidural abscess in the thoracic spine were observed. Urgent surgery was performed and the patient was treated medically with hydroxychloroquine and Favipiravir for COVID-19. Calculated antibiotic treatment with meropenem and vancomycin. No COVID-19 was detected in the abscess samples. The patient passed away later due to multiorgan failure.

Conclusion: Spondylodiscitis with spinal abscess secondary to COVID-19 infection is a severe condition and might be a consequence of dysbalanced immune response with immunodepression after COVID-19 infection.

\section{*Corresponding Author:}

Sajjad Muhammad, MD, PhD.

Address: Department of Neurosurgery, Medical Faculty, Heinrich-Heine-University, Düsseldorf, Germany

Tel: + 49 (211) 8107823

E-mail: sajjad.muhammad@med.uni-duesseldorf.de 


\section{Highlights}

- COVID-19 is the most important health problem of today.

- Spondylodiscitis with spinal abscess secondary to COVID-19 infection can be a severe condition due to dysbalanced immune response with immunodepression following infection with COVID-19.

\section{Plain Language Summary}

SARS-CoV-2 is currently a major health problem. COVID-19 infection leads to cardiovascular and neurological complications that cause additional morbidity and mortality. Spondylodiscitis with spinal abscess after CVOID-19 infection has not been reported. We report on a case of a 71-year-old man who presented with tachypnea and cough since 24 hours before admission. He was febrile and the chest CT showed a typical picture of bilateral covid-19 pneumonia. Nasopharyngeal swab demonstrated a positive PCR for COVID-19. The patient was admitted to the Intensive Care Unit (ICU) due to low oxygen saturation. Forty-eight hours after admission, the patient started to develop paraparesis which rapidly progressed to paraplegia within a few hours. MRI scan showed multi-level spondylodiscitis and epidural abscess in the thoracic spine. Urgent surgery was performed and the patient was treated medically with hydroxychloroquine and Favipiravir for COVID-19. Calculated antibiotic treatment with meropenem and vancomycin. The condition of the patient deteriorated. And he passed away due to multiorgan failure. In short, spondylodiscitis with spinal abscess secondary to COVID-19 infection is a severe condition and might be a consequence of dysbalanced immune response with immunodepression after COVID-19 infection.

\section{Background and Importance}

he number of patients infected with the

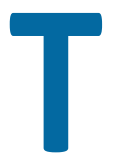
SARC-CoV-2 virus has been increasing dramatically. This disease has already infected over 20 million individuals with more than 735,000 deaths. The COVID-19 disease has been declared as a pandemic by the World health organization. In December 2019 this disease originated in Wuhan, China. With the rapid spread, in January 2020 it reached Japan, and in February/March, infected patients were reported in Europe. The virus spread briskly to North America from mid of March 2020 and the number of newly diagnosed COVID-19 patients shows the fastest growth rate. The novel coronavirus 2019 (COVID-19), which can infect both humans and animals [1, 2] has been identified as a result of intensive epidemiological and biological research. COVID-19 is a respiratory infection in nature and it can cause common cold with mild symptoms to Severe Acute Respiratory Syndrome (SARS) with respiratory failure $[3,4]$. The typical symptoms of COVID-19 infection are fever, fatigue, dry cough, and shortness of breath $[2,4,5]$ but including the cardiovascular system and gastrointestinal tract [6] it can also infect other organs. It can cause significant additional mortality in COVID-19 infected patients, spreading to other organs especially the cardiovascular system [7]. During the
COVID-19 infection, the involvement of the central nervous system is not well known. Recently, in a COVID-19 positive patient encephalopathy was reported showing the virus having possible access to the brain and involvement of the central nervous system [8]. This is among the first reports showing spondylodiscitis with spinal abscess after COVID-19 infection.

\section{Case Presentation}

A 71-year-old man without any other comorbidities presented with tachypnea and cough for a week, urinary retention, and confusion since 24 hours before admission. He was febrile and his chest CT- showed a typical picture of bilateral COVID-19 pneumonia (Figure 1, A, B). Nasopharyngeal swab displayed a positive PCR for COVID-19. The patient was admitted to the ICU due to low oxygen saturation. Forty-eight hours after admission, the patient started to develop paraparesis which rapidly progressed to paraplegia within a few hours. MRI scan was performed showing multilevel spondylodiscitis and epidural abscess in the thoracic spine (Figure 1 C, D, E).

Urgent surgery was performed to evacuate the abscess and acquire samples for microbiology. The patient was treated medically with hydroxychloroquine and Favipiravir. Calculated antibiotic treatment with meropenem and vancomycin was started immediately after the sampling. No COVID-19 was detected in the abscess 
samples. No bacterial growth during the first week of cultures was observed. After the operation, the patient's condition deteriorated fast. He passed away two days after surgery due to multiorgan failure.

\section{Discussion}

Although Talamonti et al. have presented similar COVID-19 patients who had developed the Spinal Epidural Abscess (SEA) [9], up to our knowledge this is the first case in the literature showing a lethal fulminant spondylodiscitis after COVID-19 infection. The patient was admitted with typical symptoms of COVID-19 infection including fever, cough, and dyspnea. Our patient's condition deteriorated rapidly so he was admitted to the ICU for respiratory support. After 48 hours of admission, the patient developed neurological deficits including paraparesis, sensory deficits, and urinary retention with a confirmed spondylodiscitis and intraspinal abscess in MRI scan. Despite surgical removal of the spinal abscess and broad-spectrum antiviral and anti-bacterial treatment, the patient passed away due to multiorgan failure later. The mechanism of the rapid development of spondylodiscitis with spinal abscesses was probably secondary to immune depression after COVID-19 infection. Viral infections are well known to induce an exaggerated inflammatory response with systemic inflammation including hypercytokinemia [10, 11]. In cases of COVID-19 infection a cytokine storm syndrome with a native immune depressed state has been reported $[12,13]$. Moreover, secondary Haemophagocyt- ic Lymphohistiocytosis (sHLH) is another condition with the hyperinflammatory syndrome and a fulminant fatal hypercytokinemia with multiorgan failure that has also been seen in viral infections in about $3.7-4.3 \%$ of adult sepsis cases. The treatment of the conditions like diabetes mellitus and hypertension increases the expression of angiotensin-converting enzyme 2 , which can potentially make the patient vulnerable to COVID-19 infection [14].

In our case, the patient showed initially hyperinflammation with elevated systemic leukocytes and then rapidly developed neurological symptoms with spinal abscess and spondylodiscitis. Both spondylodiscitis and spinal abscess are seen in patients with immunodepression with comorbid conditions. As our patient initially developed symptoms of COVID-19 and later he developed paraparesis and SEA, therefore, we believe that clinical course is highly suggestive for the rapid development of spondylodiscitis with spinal abscesses probably secondary to immunodepression and perhaps a bacterial superinfection. However, in the samples taken during the surgery, we did not detect any viral or bacterial presence that is also often the case in clinical practice. Moreover, due to rapid clinical decline and death of the patient, we could not analyze the immune status of the patient in detail. The ultimate diagnosis of an abscess is based on clinical, radiological, and intra-operative findings. To find the exact mechanism of secondary haemophagocytic lymphohistiocytosis and immunodepression leading to lethal condition with spondylodiscitis and spinal abscess with

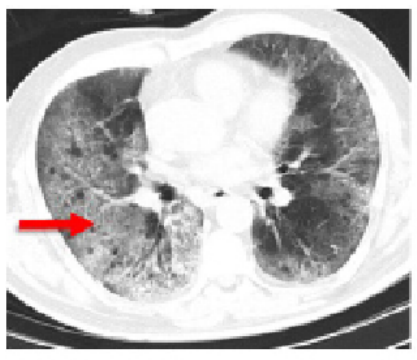

A

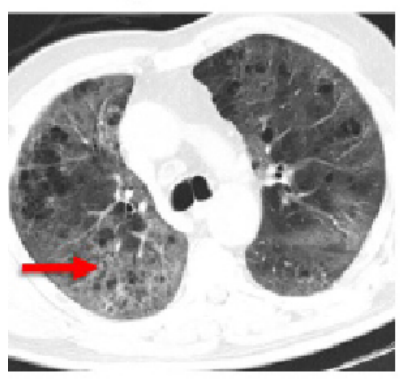

B



c

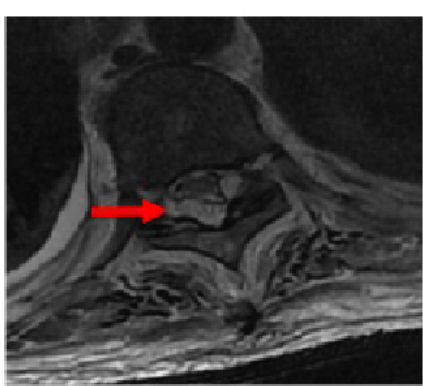

D

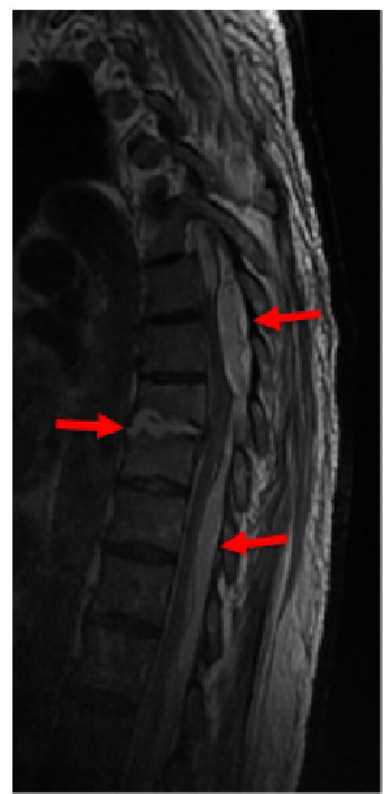

E

Figure 1. The Chest CT of the bilateral COVID-19 pneumonia

A, B: COVID-19 infection with pneumonia; and C, D, E: Discitis with multilevel abscess. 
multiorgan failure, detailed immunological investigations are required.

\section{Conclusion}

Spondylodiscitis with spinal abscess secondary to COVID-19 infection is a severe condition which can be a consequence of dysbalanced immune response with immunodepression after COVID-19 infection.

\section{Ethical Considerations}

\section{Compliance with ethical guidelines}

All ethical principles are considered in this article. The participants were informed of the purpose of the research and its implementation stages. They were also assured about the confidentiality of their information and were free to leave the study whenever they wished, and if desired, the research results would be available to them. A written consent has been obtained from the subjects. principles of the Helsinki Convention was also observed.

\section{Funding}

This research did not receive any grant from funding agencies in the public, commercial, or non-profit sectors.

\section{Authors' contributions}

Conception and design: Sajjad Muhammad; Data collection: Soheil Naderi, Nima Ostadrahimi, Abdolreza Sheikhrezai; Data analysis and interpretation: Sajjad Muhammad; Drafting the article: Sajjad Muhammad Critically revising the article: Sajjad Muhammad, Daniel Hänggi; Reviewing submitted version of manuscript: Sajjad Muhammad, Daniel Hänggi; Approving the final version of the manuscript: Soheil Naderi, Nima Ostadrahimi, Abdolreza Sheikhrezai, Sajjad Muhammad, Daniel Hänggi.

\section{Conflict of interest}

The authors declared no conflict of interest.

\section{Acknowledgements}

We acknowledge the funding support from EANS to SM.

\section{References}

[1] Zhang T, Wu Q, Zhang Zh. Probable pangolin origin of SARS-CoV-2 associated with the COVID-19 outbreak. Cur- rent Biology. 2020; 30(8):1578. [DOI:10.1016/j.cub.2020.03.063] [PMID] [PMCID]

[2] Zhou F, Yu T, Du R, Fan G, Liu Y, Liu Zh, et al. Clinical course and risk factors for mortality of adult inpatients with COVID-19 in Wuhan, China: A retrospective cohort study. The Lancet. 2020; 395(10229):1054-62. [DOI:10.1016/S01406736(20)30566-3]

[3] Guan WJ, Ni ZY, Hu Y, Liang WH, Ou CQ, He JX, et al. Clinical characteristics of coronavirus disease 2019 in China. The New England Journal of Medicine. 2020; 382:1708-20. [DOI:10.1056/NEJMoa2002032]

[4] Wang D, Hu B, Hu Ch, Zhu F, Liu X, Zhang J, et al. Clinica characteristics of 138 hospitalized patients with 2019 novel coronavirus-infected pneumonia in Wuhan, China. JAMA. 2020; 323(11):1061-9. [DOI:10.1001/jama.2020.1585] [PMID] [PMCID]

[5] Xu Zh, Shi L, Wang Y, Zhang J, Huang L, Zhang Ch, et al. Pathological findings of COVID-19 associated with acute respiratory distress syndrome. The Lancet Respiratory Medicine. 2020; 8(4):420-2. [DOI:10.1016/S2213-2600(20)30076-X]

[6] Gu J, Han B, Wang J. COVID-19: Gastrointestinal Manifestations and Potential Fecal-Oral Transmission. Gastroenterology. 2020; 158(6):1518-9. [DOI:10.1053/j.gastro.2020.02.054] [PMID] [PMCID]

[7] Guo T, Fan Y, Chen M, Wu X, Zhang L, He T, et al. Cardiovascular implications of fatal outcomes of patients with coronavirus disease 2019 (COVID-19). JAMA Cardiology. 2020; 5(7):8118.[DOI:10.1001/jamacardio.2020.1017] [PMID] [PMCID]

[8] Filatov A, Sharma P, Hindi F, Espinosa PS. Neurological complications of coronavirus disease (COVID-19): Encephalopathy. Cureus. 2020; 12(3):e7352. [DOI:10.7759/cureus.7352]

[9] Talamonti G, Colistra D, Crisà F, Cenzato M, Giorgi P, D'Aliberti G. Spinal epidural abscess in COVID-19 patients. Journal of Neurology. 2020; 1-7. [DOI:10.1007/s00415-02010211-z] [PMID] [PMCID]

[10] Muhammad S, Haasbach E, Kotchourko M, Strigli A, Krenz A, Ridder DA, et al. Influenza virus infection aggravates stroke outcome. Stroke. 2011; 42(3):783-91. [DOI:10.1161/ STROKEAHA.110.596783] [PMID]

[11] Muhammad S, Planz O, Schwaninger M. Increased plasma matrix metalloproteinase-9 levels contribute to intracerebral hemorrhage during thrombolysis after concomitant stroke and influenza infection. Cerebrovascular Diseases Extra 2016; 6(2):50-9. [DOI:10.1159/000447750] [PMID] [PMCID]

[12] Mehta P, McAuley DF, Brown M, Sanchez E, Tattersall RS, Manson JJ, HLH Across Speciality Collaboration, UK. COVID-19: Consider cytokine storm syndromes and immunosuppression. The Lancet. 2020; 395(10229):1033-4. [DOI:10.1016/ S0140-6736(20)30628-0]

[13] Siddiqi HK, Mehra MR. COVID-19 illness in native and immunosuppressed states: A clinical-therapeutic staging proposal. The Journal of Heart and Lung Transplantation. 2020; 39(5):4057.[DOI:10.1016/j.healun.2020.03.012] [PMID] [PMCID]

[14] Deravi N, Fathi M, Vakili K, Yaghoobpoor Sh, Pirzadeh $\mathrm{M}$, Mokhtari M, et al. SARS-CoV-2 infection in patients with diabetes mellitus and hypertension: A systematic review. Reviews in Cardiovascular Medicine. 2020; 21(3):385-97. [DOI:10.31083/j.rcm.2020.03.78] [PMID] 